\title{
Imeglimin, a novel, first in-class, blood glucose-lowering agent: a systematic review and meta-analysis of clinical evidence
}

\author{
THOMAS SJ CRABTREE, ${ }^{1,2}$ RALPH A DEFRONZO, ${ }^{3}$ ROBERT EJ RYDER, ${ }^{2}$ CLIFFORD J BAILEY4
}

\begin{abstract}
Imeglimin is a novel, first in-class, blood glucose-lowering agent which acts via a mitochondrial mechanism to enhance glucose-induced insulin secretion, decrease hepatic glucose output and increase glucose uptake by skeletal muscle. A systematic review and meta-analysis of randomised controlled clinical trials (RCTs) with imeglimin in adults with type 2 diabetes was undertaken. Of $\mathbf{4 5}$ articles identified, five were RCTs but, due to the format of the data, only three could be combined in a meta-analysis (total $n=180$ participants). A random-effects model found that imeglimin 1500 mg twice daily as monotherapy and add-on to metformin or sitagliptin was associated with reductions of $\mathrm{HbA}_{1 \mathrm{c}}$ by $-0.63 \%(95 \% \mathrm{Cl}-0.84$ to -0.42$)(-6.6 \mathrm{mmol} / \mathrm{mol}, 95 \% \mathrm{Cl}-8.8$ to -4.4 ) and reductions of fasting plasma glucose by -0.52 $\mathrm{mmol} / \mathrm{L}(95 \% \mathrm{Cl}-0.80$ to -0.24$)$ compared with placebo. Adverse events were minimal, mostly gastrointestinal, and without hypoglycaemia. It is concluded that imeglimin displays promising improvements in $\mathrm{HbA}_{1 \mathrm{c}}$ and fasting plasma glucose and is generally well tolerated.

Br J Diabetes 2020;20:28-31
\end{abstract}

Key words: type 2 diabetes; mitochondria; imeglimin; glimins; tetrahydrotriazine, systematic review, meta-analysis

\section{Introduction}

Type 2 diabetes is the product of multiple pathogenic factors including insulin resistance, beta-cell dysfunction and many other disturbances that underlie the development of hyperglycaemia. ${ }^{1}$ Current glucose-lowering medications taken as monotherapy or in combinations are often unable to reinstate normoglycaemia,

University Hospitals of Derby and Burton NHS Trust; University of Nottingham; Sandwell and West Birmingham Hospitals NHS Trust, UK

2 City Hospital, Birmingham, UK

University of Texas Health Science Center, San Antonio, Texas, USA

${ }^{4}$ Life and Health Sciences, Aston University, Birmingham, UK

Address for correspondence: Dr Thomas SJ Crabtree Department of Diabetes, Royal Derby Hospital, Uttoxeter Road, Derby, DE22 3NE

E-mail: t.crabtree@nhs.net

https://doi.org/10.15277/bjd.2020.247 indicating the need for new therapies with different modes of action. This paper focuses on imeglimin, a new type of glucoselowering agent (a glimin) continuing after delays in phase 3 development for the treatment of type 2 diabetes.

Imeglimin is a tetrahydrotriazine that acts on mitochondria to increase flux through complex II of the respiratory chain, increasing ATP synthesis and reducing the production of reactive oxygen species. ${ }^{2}$ Mitochondrial dysfunction has been shown to play an important pathogenic role in the development of type 2 diabetes, ${ }^{3}$ and can be demonstrated in the normal, glucosetolerant, insulin-resistant offspring of diabetic parents. ${ }^{4}$ Studies in animal models have shown that imeglimin impacts the pathophysiology of type 2 diabetes mellitus by improving glucoseinduced insulin secretion, increasing beta-cell mass, decreasing hepatic glucose output and increasing skeletal muscle glucose uptake. ${ }^{2,5-8}$ Imeglimin may also protect endothelial cells from the effects of glucotoxicity with potential beneficial cardiovascular effects. ${ }^{9-11}$

This systematic review and meta-analysis assesses the evidence from clinical trials of imeglimin compared with placebo or other established oral glucose-lowering drugs.

\section{Methods}

The word "imeglimin" was searched using the Ovid Medline and Embase databases and grey literature. Randomised controlled trials or cluster randomised controlled trials conducted with imeglimin against placebo and/or other oral glucose-lowering agents in human subjects were considered for inclusion. There were no restrictions of language or timeframe. Primary outcomes of interest for meta-analysis (where quantity and quality of data allowed) were $\mathrm{HbA}_{1 \mathrm{c}}$ and fasting plasma glucose (FPG) following treatment with imeglimin. Secondary outcomes included adverse events and hypoglycaemia. Identified publications were assessed independently by two reviewers. A third reviewer was available but not required to adjudicate any disagreement over inclusions/exclusions. References listed in identified publications were cross-checked for any publications missed by the initial searches. All identified randomised controlled trials were assessed using the Cochrane risk of bias assessment. Data were extracted using Cochrane data collection forms. Outcomes of interest underwent random-effects analysis using RevMan 5.3. The review protocol was registered with PROSPERO prior to commencement (CRD42019155733). 
Figure 1. Flow chart showing the search and screening process of the systematic review

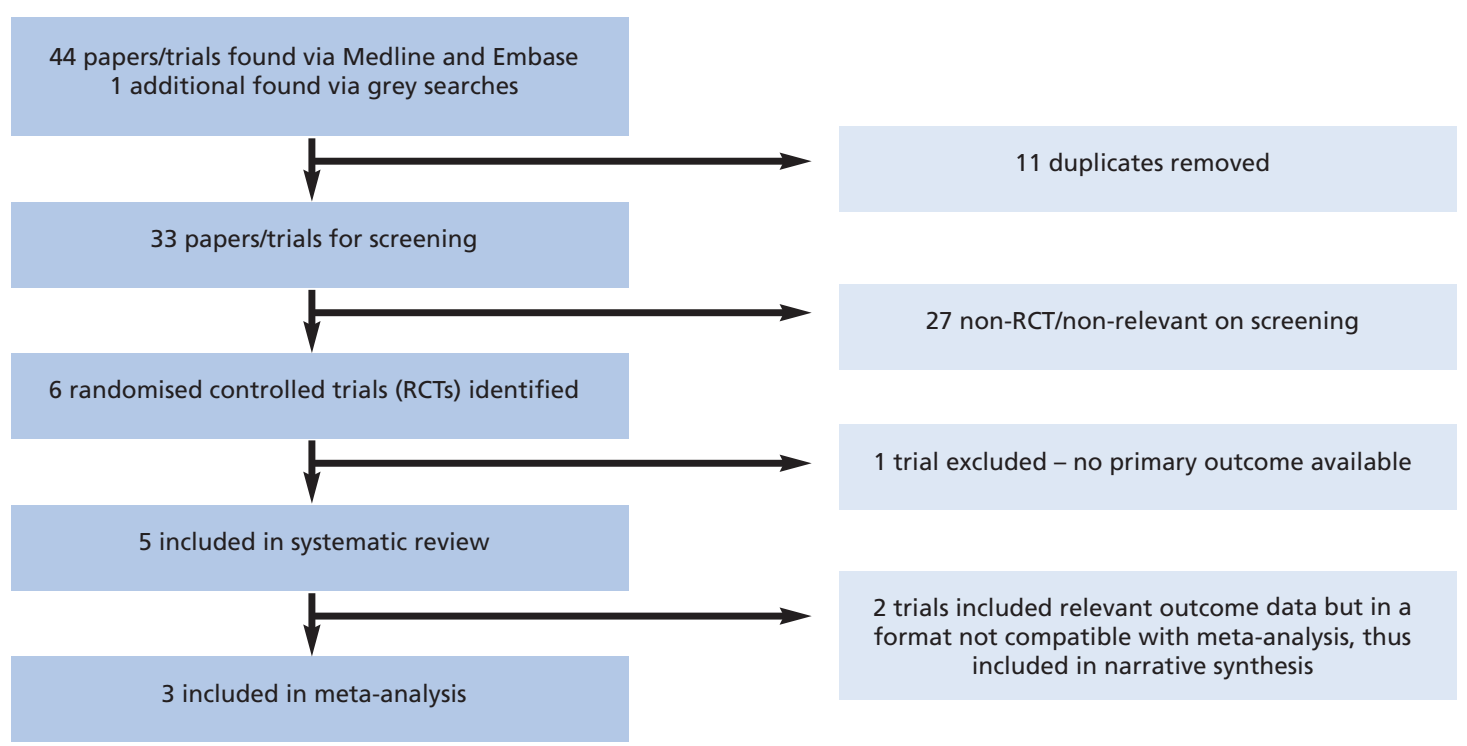

\section{Results}

Of 45 articles identified by initial searches, three randomised controlled trials were accepted for meta-analysis of the primary outcomes of $\mathrm{HbA}_{1 \mathrm{c}}$ and FPG (Figure 1). Two further identified studies were not included in the meta-analysis due to their use of placebo subtracted values rather than mean and SD for the individual arms. These will be discussed in the narrative. Baseline characteristics, study size, dose of imeglimin and follow-up of the five identified studies are shown in Table 1.

\section{Primary outcomes}

Baseline mean $\pm \mathrm{SD}$ values for $\mathrm{HbA}_{1 \mathrm{c}}$ and FPG in the three included studies were $8.2 \pm 0.6 \%(66 \pm 7 \mathrm{mmol} / \mathrm{mol})$ and $10.3 \pm 1.9$ $\mathrm{mmol} / \mathrm{L}$, respectively. The meta-analyses of $\mathrm{HbA} 1 \mathrm{c}$ and FPG data from the three included studies are shown in Figure 2, along with risk of bias assessment for these studies. ${ }^{12-14}$ The randomeffects model determined that imeglimin $1500 \mathrm{mg}$ twice daily was associated with reductions of $\mathrm{HbA}_{1 \mathrm{c}}$ of $-0.63 \%(95 \% \mathrm{Cl}$ -0.84 to -0.42$)(-6.6 \mathrm{mmol} / \mathrm{mol}, 95 \% \mathrm{Cl}-8.8$ to -4.4$)$ and reductions of FPG by $-0.52 \mathrm{mmol} / \mathrm{L}(95 \% \mathrm{Cl}-0.80$ to -0.24$)$ compared with placebo.

Two studies not included in the meta-analysis showed significant placebo-subtracted decreases in $\mathrm{HbA}_{1 \mathrm{c}}$ and FPG with imeglimin.15 16 In a 24-week phase 2b monotherapy trial with 299 Japanese patients, imeglimin (1500 mg twice daily) was associated with placebo-corrected reductions of $\mathrm{HbA}_{1 \mathrm{c}}$ (by $-1.0 \%)(-11 \mathrm{mmol} / \mathrm{mol})$ and FPG (by $-1.4 \mathrm{mmol} / \mathrm{L})$. In the recent 24-week phase 3 monotherapy trial, placebo-corrected reductions in $\mathrm{HbA}_{1 \mathrm{c}}$ and $\mathrm{FPG}$ amongst 213 participants were $-0.87 \%$ (-9 $\mathrm{mmol} / \mathrm{mol}$ ) and $-1.1 \mathrm{mmol} / \mathrm{L}$, respectively, with imeglimin (1000 mg twice daily). Each of these results was significant to $p<0.001$, but the confidence intervals or variances were not reported.

\section{Secondary outcomes}

Numerically fewer treatment-emergent adverse events were noted with imeglimin compared with placebo in two of the three included studies, ${ }^{12,14}$ and a small increase (3.9\%) was noted in the other study. ${ }^{13}$ None of these events was considered serious, and the most common side effects were gastrointestinal, affecting $\leq 6 \%$ of patients taking imeglimin where reported. ${ }^{12,14}$ No adverse cardiovascular events were noted.

Hypoglycaemia (severity not defined) was noted on four occasions in one study during the run-in phase (ie, before commencing imeglimin), ${ }^{14}$ but no hypoglycaemia events were identified with imeglimin in this or any of the other studies. One study noted a 'slight decrease' in weight when participants received imeglimin in combination with metformin compared with metformin alone. ${ }^{13}$ No other studies reported body weight outcome.

\section{Discussion}

The present meta-analysis confirms that the novel glucoselowering agent imeglimin consistently reduced $\mathrm{HbA}_{1 \mathrm{c}}$ and $\mathrm{FPG}$ during randomised controlled trials in type 2 diabetes patients when used either as monotherapy or add-on to metformin or sitagliptin. Overall reductions of $\mathrm{HbA}_{1 \mathrm{c}}$ (by $0.63 \%$ ) and FPG (by $0.52 \mathrm{mmol} / \mathrm{L}$ ) with the $1500 \mathrm{mg}$ twice daily dose of imeglimin are comparable with efficacy data reported for some other classes of glucose-lowering agents such as dipeptidyl peptidase4 inhibitors and sodium-glucose co-transporter-2 inhibitors. ${ }^{17}$

Adverse effects were mostly minor and related to the gastrointestinal tract, indicating that imeglimin was generally well tolerated. Severe hypoglycaemia was not identified with imeglimin, consistent with agents that exhibit similar glucoselowering efficacy. ${ }^{17}$ However, evidence from larger studies will be required to confirm this. Further evidence of the effect of imeglimin on body weight is also needed. 
Table 1 Characteristics and main findings of randomised controlled studies identified from systematic searches assessing imeglimin versus placebo included in systematic review

\begin{tabular}{|c|c|c|c|c|}
\hline Study & Design & Participants & Arms & Results \\
\hline Pirags et al, $2012^{12}$ & $\begin{array}{l}\text { 8-week, multicentre, randomised, } \\
\text { four-arm parallel group study. } \\
\text { Patients on no other therapy }\end{array}$ & $\begin{array}{l}\mathrm{n}=95 \\
\text { Mean age in imeglimin group } \\
1500 \mathrm{mg} \text { BD group } 60.0 \text { years } \\
\text { Mean BMI in imeglimin group } \\
32.2 \mathrm{~kg} / \mathrm{m}^{2} \\
\text { All arms were broadly similar }\end{array}$ & $\begin{array}{l}\text { Patients randomised to } \\
\text { imeglimin } 500 \text { mg BD; } \\
\text { imeglimin } 1500 \text { mg BD; } \\
\text { metformin } 850 \text { mg BD or } \\
\text { placebo }\end{array}$ & $\begin{array}{l}\text { Imeglimin } 1500 \mathrm{mg} \mathrm{BD} \\
\text { Changed } \mathrm{HbA} \mathrm{A}_{1 \mathrm{c}}-0.18 \% \text { vs }+0.31 \% \\
\text { in placebo } \mathrm{Changed} \mathrm{FPG}-1.02 \mathrm{mmol} / \mathrm{L} \\
\text { vs }+0.78 \mathrm{mmol} / \mathrm{L} \text { in placebo } \\
\text { Fewer adverse events with imeglimin } \\
\text { vs placebo } \\
\text { Adverse events mostly Gl }\end{array}$ \\
\hline Fouqueray et al, $2013^{13}$ & $\begin{array}{l}\text { 12-week, multicentre, } \\
\text { randomised, double-blind, placebo } \\
\text { controlled, parallel group study. } \\
\text { Patients inadequately controlled } \\
\text { on maximum dose of metformin }\end{array}$ & $\begin{array}{l}\mathrm{n}=156 \\
\text { Age range } 18-70 \text { years } \\
\text { BMI not reported }\end{array}$ & $\begin{array}{l}\text { Patients randomised to } \\
\text { combination therapy with } \\
\text { metformin }+ \text { imeglimin } \\
1500 \text { mg BD vs placebo + } \\
\text { metformin }\end{array}$ & $\begin{array}{l}\text { Imeglimin } 1500 \mathrm{mg} \mathrm{BD} \\
\text { Changed } \mathrm{HbA}_{1 \mathrm{c}}-0.65 \% \text { vs }-0.21 \% \\
\text { in placebo } \\
\text { Changed FPG }-0.91 \mathrm{mmol} / \mathrm{L} \text { vs } \\
+0.36 \mathrm{mmol} / \mathrm{L} \text { in placebo } \\
\text { Numerical increase ( } 3.9 \% \text { ) of patients } \\
\text { with adverse events with imeglimin } \\
\text { vs placebo } \\
\text { 'Slight decrease' in body weight } \\
\text { observed }\end{array}$ \\
\hline Fouqueray et al, 2014 ${ }^{14}$ & $\begin{array}{l}\text { 12-week, multicentre, } \\
\text { randomised, double-blind, placebo } \\
\text { controlled, parallel group study. } \\
\text { Patients inadequately controlled } \\
\text { on sitagliptin alone. }\end{array}$ & $\begin{array}{l}\mathrm{n}=170 \\
\text { Age range } 18-75 \text { years } \\
\text { BMl } 20-40 \mathrm{~kg} / \mathrm{m}^{2}\end{array}$ & $\begin{array}{l}\text { Patients randomised to } \\
\text { combination therapy with } \\
\text { sitagliptin + imeglimin } \\
1500 \text { mg BD vs placebo + } \\
\text { sitagliptin }\end{array}$ & $\begin{array}{l}\text { Imeglimin } 1500 \mathrm{mg} \mathrm{BD} \\
\text { Changed } \mathrm{HbA} \mathrm{A}_{1 \mathrm{c}}-0.6 \% \text { vs }+0.12 \% \text { in } \\
\text { placebo } \\
\text { Changed FPG }-0.93 \mathrm{mmol} / \mathrm{L} \text { vs } \\
-0.11 \mathrm{mmol} / \mathrm{L} \text { in placebo } \\
\text { Fewer adverse events with imeglimin } \\
\text { vs placebo }\end{array}$ \\
\hline Dubourg et al, 2017 $15 *$ & $\begin{array}{l}\text { 24-week, multicentre, } \\
\text { randomised, double-blind, placebo } \\
\text { controlled trial. Japanese patients } \\
\text { on no other therapy. }\end{array}$ & $\begin{array}{l}n=299 \\
\text { Mean age in imeglimin group } \\
1500 \mathrm{mg} \text { BD group } 57.6 \text { years } \\
\text { Mean BMI in imeglimin } 1500 \mathrm{mg} \\
\text { BD group } 26.8 \mathrm{~kg} / \mathrm{m}^{2} \\
\text { All arms broadly similar }\end{array}$ & $\begin{array}{l}\text { Patients were randomised } \\
\text { to imeglimin } 500 \text { mg BD, } \\
\text { imeglimin } 1000 \mathrm{mg} \mathrm{BD} \text {, } \\
\text { imeglimin } 1500 \mathrm{mg} \mathrm{BD} \text { or } \\
\text { placebo }\end{array}$ & $\begin{array}{l}\text { Imeglimin } 1500 \mathrm{mg} \text { BD } \\
\text { Placebo corrected reduction in } \\
\mathrm{HbA}_{1 \mathrm{c}}-1.0 \% \\
\text { Placebo corrected reduction in } \\
\text { FPG }-1.4 \mathrm{mmol}\end{array}$ \\
\hline $\begin{array}{l}\text { TIMES } 1 \text { trial }^{16 *} \\
\text { Topline results as } \\
\text { per Poxel Website }\end{array}$ & $\begin{array}{l}\text { 24-week, multicentre, } \\
\text { randomised, double-blind, placebo } \\
\text { controlled trial. Japanese patients } \\
\text { on no other therapy }\end{array}$ & $\begin{array}{l}\mathrm{n}=213 \\
\text { Baseline characteristics not } \\
\text { available }\end{array}$ & $\begin{array}{l}\text { Patients were randomised } \\
\text { to imeglimin } 1000 \mathrm{mg} \mathrm{BD} \\
\text { or placebo }\end{array}$ & $\begin{array}{l}\text { Imeglimin } 1000 \mathrm{mg} \text { BD } \\
\text { Placebo corrected reduction in } \\
\mathrm{HbA}_{1 c}-0.87 \% \\
\text { Placebo corrected reduction in } \\
\text { FPG }-1.1 \mathrm{mmol} / \mathrm{L}\end{array}$ \\
\hline
\end{tabular}

Figure 2. Forest plot of meta-analysis of imeglimin versus placebo for (a) $\mathrm{HbA}_{1 \mathrm{c}}(\%)$ and (b) fasting plasma glucose (mmol/L) using a random-effect model. Risk of bias assessment is included

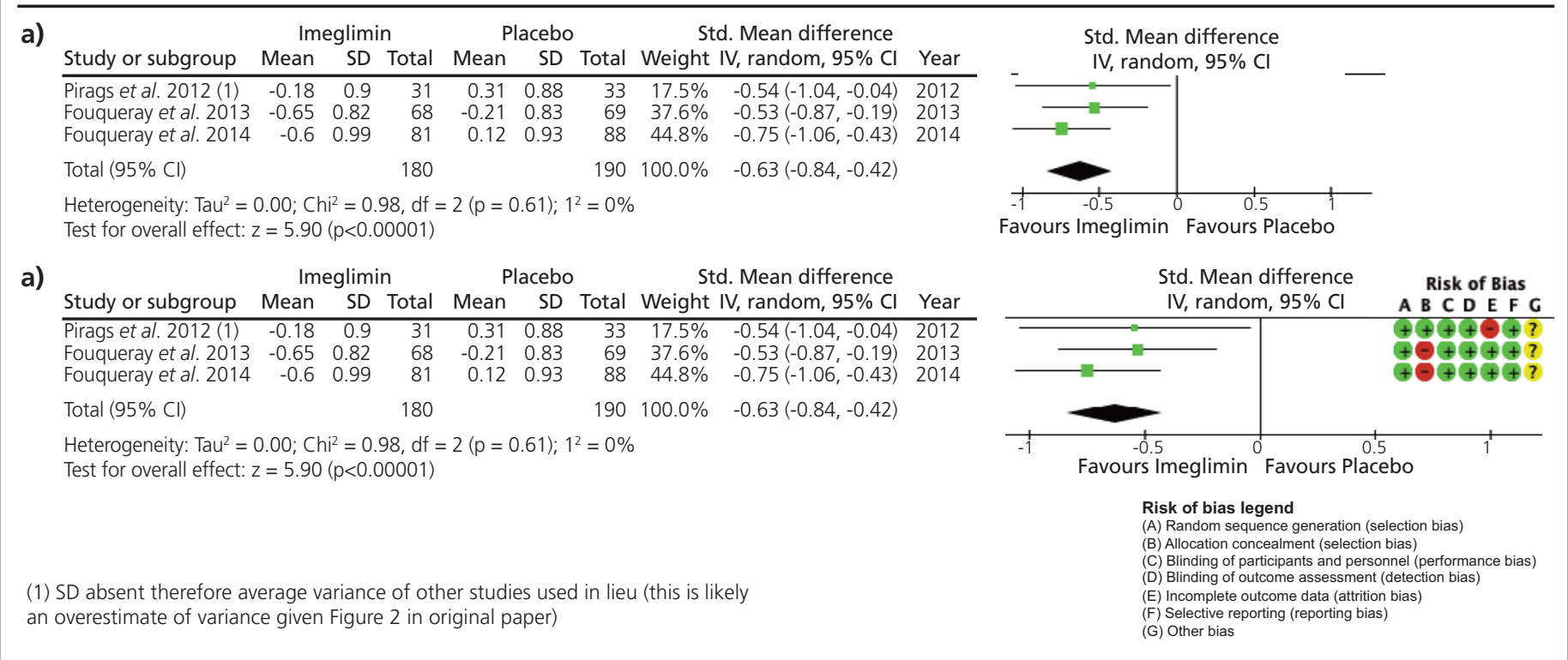




\section{Key messages}

- Imeglimin is a novel, first in-class, glucose-lowering agent for the management of type 2 diabetes

- Imeglimin acts via a mitochondrial mechanism to increase glucose uptake by skeletal muscle, decrease hepatic glucose output and increase glucosedependent insulin secretion

- Initial clinical trials show that imeglimin reduces $\mathrm{HbA}_{1 \mathrm{c}}$ and fasting plasma glucose in type 2 diabetes, and is generally well tolerated

The bias assessment noted insufficient information about the allocation process and incomplete data that precluded more extensive analyses. Other limitations concerned the modest numbers of patients, the duration of the trials (longest 24 weeks) and the need for studies that assess the effects of imeglimin in different groups of type 2 diabetes patients. These should consider different ethnicities and co-morbidities, different stages of disease progression and different combinations of agents, including measures of long-term efficacy and safety.

These initial trials indicate that imeglimin exerts a significant glucose-lowering effect as monotherapy or in combination with metformin or sitagliptin, achieving comparable efficacy with some other classes of glucose-lowering agents. Imeglimin was well tolerated and showed an acceptable safety profile in studies to date. Larger, longer and more detailed trials are awaited to expand present information.

Conflict of interest TC has nothing to disclose. RAD Advisory Board: AstraZeneca, Novo Nordisk, Janssen, Boehringer-Ingelheim, Intarcia, Poxel Honorarium. Research Support: Boehringer-Ingelheim, AstraZeneca, Janssen, Merck - Research Grant - (Investigator). Speaker's Bureau: NovoNordisk, AstraZeneca - Honorarium (Speaker). REJR: speaker fees, and/or consultancy fees and/or educational sponsorships from AstraZeneca, BioQuest, GI Dynamics, Janssen and Novo Nordisk. CB reports personal fees from Poxel, outside the submitted work.

\section{Funding None.}

\section{References}

1. DeFronzo RA, Ferrannini E, Groop L, et al. Type 2 diabetes mellitus. Nat Rev Dis Primers 2015;1:15019. https://doi.org/10.1038/nrdp.2015.19

2. Vial G, Chauvin M-A, Bendridi N, et al. Imeglimin normalizes glucose tolerance and insulin sensitivity and improves mitochondrial function in liver

of a high-fat, high-sucrose diet mice model. Diabetes 2015;64:2254-64. https://doi.org/10.2337/db14-1220

3. Patti M-E, Corvera $S$. The role of mitochondria in the pathogenesis of type 2 diabetes. Endocr Rev 2010;31:364-95. https://doi.org/10.1210/er.2009-0027

4. Petersen KF, Dufour S, Befroy D, et al. Impaired mitochondrial activity in the insulin-resistant offspring of patients with type 2 diabetes. $N$ Engl $J$ Med 2004;350:664-71. https://doi.org/10.1056/NEJMoa031314

5. Li J, Shirakawa, Y, Togashi T, et al. Effects of imeglimin on insulin secretion, beta-cell proliferation, or apoptosis in mouse islets. J Diabetes Invest 2018;9:12. https://doi.org/10.1111/jdi.12937

6. Pacini G. Mari A, Fouqueray P, et al. Imeglimin increases glucose-dependent insulin secretion and improves beta-cell function in patients with type 2 diabetes. Diabetes Obes Metab 2015;17:541-5. https://doi.org/10.1111/dom.12452

7. Hallakou-Bozec S, Kergoat M, Bolze S, Lebovitz HE. Imeglimin preserves beta cell function and mass in male zucker diabetic fatty rats. Endocr Pract 2017; 23:1. https://d1 io3yog0oux5.cloudfront.net_938fdfc18803a883031931f148 c6cae6/poxelpharma/db/419/2331/file/2016-11-17_WCIRDC+Imeglimin+ beta+cell+function+and+mass+Final.pdf

8. Lablanche S, Tubbs E, Cottet-Rousselle C, et al. Imeglimin protects INS-1 cells and human islets against high glucose- and high fructose-induced cell death by inhibiting the mitochondrial PTP opening. Diabetes 2018;67(Suppl 1):81-OR. https://doi.org/10.2337/db18-81-OR

9. Detaille D, Vial G, Borelet A-L, et al. Imeglimin prevents human endothelial cell death by inhibiting mitochondrial permeability transition without inhibiting mitochondrial respiration. Cell Death Discovery 2016;2:15072. https://doi.org/10.1038/cddiscovery.2015.72

10. Lachaux M, Nicol L, Hamzaou M, et al. Imeglimin protects from diabetic cardiomyopathy in the obese zucker rat. Diabetes 2017;66(Suppl 1):2054-P.

11. Lachaux M, Souille M, Remy-Jouet I, et al. Acute imeglimin treatment improves metabolic syndrome-related cardiac and coronary endothelial dysfunction in the zucker fa/fa rat. Eur Heart J 2018;39:484-5.

12. Pirags $V$, Lebovitz $H$, Fouqueray P. Imeglimin, a novel glimin oral antidiabetic, exhibits a good efficacy and safety profile in type 2 diabetic patients. Diabetes Obes Metab 2012;14:852-8. https://doi.org/10.1111/j.1463-1326.2012.01611.x

13. Fouqueray $P$, Pirags $V$, Inzucchi $S E$, et al. The efficacy and safety of imeglimin as add-on therapy in patients with type 2 diabetes inadequately controlled with metformin monotherapy. Diabetes Care 2013;36:565-68. https://doi.org/10.2337/dc12-0453

14. Fouqueray $P$, Pirags $V$, Diamant $M$, et al. The efficacy and safety of imeglimin as add-on therapy in patients with type 2 diabetes inadequately controlled with sitagliptin monotherapy. Diabetes Care 2014;37:1924-30. https://doi.org/10.2337/dc13-2349

15. Dubourg J, Ueki $\mathrm{K}$, Watada $\mathrm{H}$, et al. Imeglimin monotherapy in Japanese patients with type 2 diabetes: results from a randomised, 24-week, double-blind, placebo-controlled, phase IIb trial. Diabetologia 2017;60(Suppl 1):843.

16. Poxel Pharma. Poxel and Sumitomo Dainippon Pharma announce positive top-line results for imeglimin phase 3 trial (TIMES 1) in Japan for the treatment of type 2 diabetes. 9 April 2019. https://www.poxelpharma.com/ en_us/news-media/press-releases/detail/117/poxel-and-sumitomo-dainippon-pharma-announce-positive (accessed 23 March 2020).

17. Bailey CJ. The current drug treatment landscape for diabetes and perspectives for the future. Clin Pharmacol Ther 2015;98:170-84. https://doi.org/10.1002/cpt.144 